\title{
TU/e EmonONEN

\section{A fluid EOQ model of perishable items with intermittent high and low demand rates}

\section{Citation for published version (APA):}

Boxma, O. J., Perry, D., \& Zacks, S. (2015). A fluid EOQ model of perishable items with intermittent high and low demand rates. Mathematics of Operations Research, 40(2), 390-402. https://doi.org/10.1287/moor.2014.0675

\section{Document license:}

TAVERNE

DOI:

10.1287/moor.2014.0675

Document status and date:

Published: 01/01/2015

\section{Document Version:}

Publisher's PDF, also known as Version of Record (includes final page, issue and volume numbers)

\section{Please check the document version of this publication:}

- A submitted manuscript is the version of the article upon submission and before peer-review. There can be important differences between the submitted version and the official published version of record. People interested in the research are advised to contact the author for the final version of the publication, or visit the $\mathrm{DOI}$ to the publisher's website.

- The final author version and the galley proof are versions of the publication after peer review.

- The final published version features the final layout of the paper including the volume, issue and page numbers.

Link to publication

\section{General rights}

Copyright and moral rights for the publications made accessible in the public portal are retained by the authors and/or other copyright owners and it is a condition of accessing publications that users recognise and abide by the legal requirements associated with these rights.

- Users may download and print one copy of any publication from the public portal for the purpose of private study or research.

- You may not further distribute the material or use it for any profit-making activity or commercial gain

- You may freely distribute the URL identifying the publication in the public portal.

If the publication is distributed under the terms of Article 25fa of the Dutch Copyright Act, indicated by the "Taverne" license above, please follow below link for the End User Agreement:

www.tue.nl/taverne

Take down policy

If you believe that this document breaches copyright please contact us at:

openaccess@tue.nl

providing details and we will investigate your claim. 


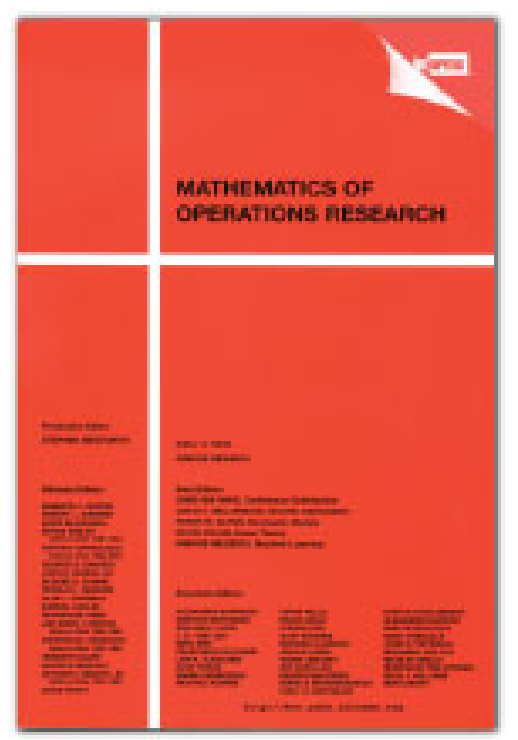

\section{Mathematics of Operations Research}

Publication details, including instructions for authors and subscription information: http:// pubsonline.informs.org

\section{A Fluid EOQ Model of Perishable Items with Intermittent High and Low Demand Rates}

Onno Boxma, David Perry, Shelley Zacks

To cite this article:

Onno Boxma, David Perry, Shelley Zacks (2015) A Fluid EOQ Model of Perishable Items with Intermittent High and Low Demand Rates. Mathematics of Operations Research 40(2):390-402. http:// dx. doi.org/ 10.1287/ moor. 2014.0675

\section{Full terms and conditions of use: http://pubsonline.informs.org/page/terms-and-conditions}

This article may be used only for the purposes of research, teaching, and/or private study. Commercial use or systematic downloading (by robots or other automatic processes) is prohibited without explicit Publisher approval, unless otherwise noted. For more information, contact permissions@informs.org.

The Publisher does not warrant or guarantee the article's accuracy, completeness, merchantability, fitness for a particular purpose, or non-infringement. Descriptions of, or references to, products or publications, or inclusion of an advertisement in this article, neither constitutes nor implies a guarantee, endorsement, or support of claims made of that product, publication, or service.

Copyright @ 2015, INFORMS

Please scroll down for article-it is on subsequent pages

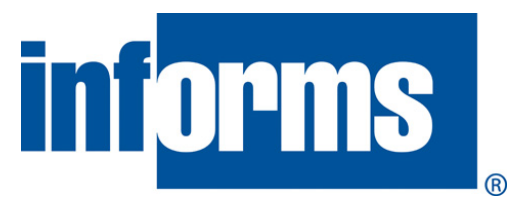

INFORMS is the largest professional society in the world for professionals in the fields of operations research, management science, and analytics.

For more information on INFORMS, its publications, membership, or meetings visit http:// www. informs.org 


\title{
A Fluid EOQ Model of Perishable Items with Intermittent High and Low Demand Rates
}

\author{
Onno Boxma \\ EURANDOM and Department of Mathematics and Computer Science, Eindhoven University of Technology, \\ 5600 MB Eindhoven, The Netherlands, o.j.boxma@tue.nl \\ David Perry \\ Department of Statistics, University of Haifa, Haifa 31905, Israel, dperry@stat.haifa.ac.il \\ Shelley Zacks \\ Department of Mathematical Sciences, Binghamton University, Binghamton, \\ New York 13902, shelly@math.binghamton.edu
}

\begin{abstract}
We consider a stochastic fluid EOQ-type model with demand rates operating in a two-state random environment. This environment alternates between exponentially distributed periods of high demand and generally distributed periods of low demand. The inventory level starts at some level $q$, and decreases linearly at rate $\beta_{H}$ during the periods of high demand, and at rate $\beta_{L}<\beta_{H}$ at periods of low demand. Refilling of the inventory level to level $q$ is required when the first of two events takes place: Either the buffer level reaches zero, or the buffer content becomes outdated. If such an event occurs during a high demand period, an order is instantaneously placed; otherwise, ordering is postponed until the beginning of the next high demand period.

We determine the steady-state distribution of the inventory level, as well as other quantities of interest such as the distribution of the time until a refill is required. Finally, for a given cost/revenue structure, we determine the long-run average profit, and we consider the problem of choosing $q$ such that the profit is optimized.

Keywords: EOQ model; perishable inventories; outdatings; unsatisfied demands; regenerative process; compound Poisson process; Laplace transform

MSC2000 subject classification: Primary: 90B05; secondary: 90B30, 60K30

OR/MS subject classification: Primary: inventory/production; probability; secondary: perishable/aging items; regenerative processes; Markov processes

History: Received February 22, 2011; revised June 9, 2012, June 11, 2013, December 1, 2013. Published online in Articles in Advance August 28, 2014.
\end{abstract}

1. Introduction. The simple Economic Order Quantity (EOQ) model for inventory control is one of the most fundamental models in Operations Research. It was first presented by Harris [6] in 1915, and was mathematically formulated by Wilson [21] in 1934. The EOQ model is the most basic of all inventory models, owing to its simple objective function that lucidly describes the trade-off between the constant set-up costs and the variable holding costs. The robustness of the Wilson Root Formula serves as an effective approximation for many intricate extensions based on the EOQ model. The trade-off effect among the different types of costs is used as the basis for many extensions of EOQ-type problems. The majority of the extensions is directed to periodic review models that were formulated as dynamic programming problems and complexity problems of combinatorial optimization. However, another main stream of extensions focuses on the performance analysis of stochastic models that operate under conditions of continuous review. This study belongs to the latter family of models.

We consider a stochastic fluid EOQ-type model of perishable items, whose shelf life equals the constant $t_{0}$. At the beginning of the cycle, a quantity $q$ is placed in stock. All items left in stock after $t_{0}$ are discarded. The system operates in a two-state random environment, alternating between a good state (i.e., a period of high demand rate) and a bad state (i.e., a period of low demand rate) according to a continuous-time semi-Markov chain. The net demand rate equals $\beta_{H}$ during the good state and $\beta_{L}$ during the bad state, where $\beta_{H}>\beta_{L}$, since the sales during good periods are higher than those during bad periods. (Note that this assumption is not necessary for the analysis). The lengths of high demand periods are independent and exponentially distributed random variables and the lengths of low demand periods are i.i.d. random variables with a general distribution; the lengths of all periods are also independent of each other.

Reordering is required when the first of two events takes place: Either the buffer level reaches zero, or the buffer content becomes outdated (perishes). If such an event occurs during a high demand period, an order is instantaneously placed. If such an event occurs during a low demand period, then ordering is postponed until the end of the low demand period. Thus the content level process is regenerative; cycles start at moments of order arrivals. In Remark 2 we comment on the assumption of postponing the order until the beginning of the next high demand period.

A controller wishes to maximize the long-run average profit. We assume that he can control the order quantity $q$. Accordingly, he wishes to select the optimal $q:=q^{*}$ so as to properly balance revenues and costs. Revenues are 
earned by selling units. The costs include set-up costs, which are incurred each time an order is placed, holding costs, the costs for discarded units (outdating), and the costs for unsatisfied demand.

REMARK 1. The two-state random environment model reflects situations in which the demand rate for a certain commodity undergoes periodically recurring changes. The demand rates for many goods go up and down between different levels according to changes in the interest rate or due to fashion or other recurring seasonal/external effects. Models including a multistate random environment can be suitable for such situations; the two-state case presented in this paper could serve as a first approximation.

The rationale behind having exponentially distributed high demand periods is that low demand periods may represent some form of recession, and as a first approximation, recessions may occur according to a Poisson process. This yields exponentially distributed high demand periods. Recessions within recessions may give rise to extended low demand periods; it seems less natural to assume that low demand periods are exponentially distributed.

Literature review. The literature about Perishable Inventory Systems (PIS) is quite rich. Over the last three decades, several comprehensive reviews have been published (Baron [1], Giri and Chaudhuri [4], Karaesmen et al. [7], Nahmias [11, 12], Raafat [16]). They reveal a strong emphasis on the design of algorithms for optimization and/or control, i.e., when to place an order and how much to order. Recently, there has been a growing interest in the stochastic analysis of perishable inventory systems that operate under some heuristic control policy; see Karaesmen et al. [7]. Our study belongs to the latter category. First, we introduce the performance analysis of a certain stochastic fluid EOQ model (the case of compound Poisson demand is introduced in Baron [1], but without the randomness of the environment). Then, a specific objective function is introduced and the functionals obtained lay the groundwork for some numerical and sensitivity analysis.

The survey of Karaesmen et al. [7] classifies continuous review models into three categories: without fixed ordering costs or lead times, without fixed ordering costs having positive lead time, and with fixed ordering costs (typically with zero lead time). Graves [5] originated the first category. He assumed that items are continuously produced and perish after a deterministic time, and that demand follows a compound Poisson process with either a single-unit or an exponential demand at each arrival. Pal [13] originated the second category, investigating the performance of an $(S-1, S)$ control policy. The third category, originated by Weiss [20], is relevant to our model; Karaesmen et al. [7], Lian and Liu [8], Lian et al. [9], Liu and Lian [10], Perry et al. [15] made significant contributions to models in this category. Lian et al. [9] consider discrete demand for items and time to perishability that is either fixed-and-known or that follows a Phase-type distribution.

There are two types of perishable inventory models. The first family of models assumes that the quality of the items is slowly decreasing over time. For an early review of work concerning this family of models see Nahmias [11]; see also Nahmias [12]. Rajan and Steinberg [17] consider perishable items with order set-up costs. They assume that the quantity of good items deteriorates at an exponential rate, and solve the problem of finding the joint optimal ordering and pricing strategy. The second family is of models with obsolescences (Song and Lau [18]). Here, the items might perish at each period with some probability that is typically increasing over time (Berk and Gürler [3], Perry [14]).

A large variety of inventory models is presented in detail in the (Zipkin [24]) monograph. The stochastic models are based on point processes for the demand arrivals in random environments (in Zipkin [24] called "world driven"). The fluid systems in Zipkin [24] are deterministic EOQ models with the classical extensions such as planned backorders, limited capacity, quantity discounts, and imperfect quality. In the deterministic setting, time-varying demands are also considered, but without multiple order quantities.

To the best of our knowledge, the stochastic EOQ model with the added features of perishability and a non-Markovian environment expounded in this paper is new. The work that is most closely related to this study is Baron et al. [2]. In fact, we generalize Baron et al. [2] by adding two important features: (i) The items stored are subject to perishability with a predetermined expiration date, viz., the constant $t_{0}$ (not a decision variable). (ii) The lengths of the bad periods of the random environment are not necessarily exponential. Our approach in the present paper is completely different.

In the sequel we model the lengths of the intermittent high and low demand periods as an alternating renewal process. Such a model can be described as a generalized integrated telegrapher process (see Zacks [22]), with a particle moving with velocities $\beta_{H}$ and $\beta_{L}$ intermittently. The distribution of the total cumulative demand, $Q(t)$, for our model can be derived as in Zacks [22]. The distribution of the inventory level, $X(t)$, can be easily derived from that of $Q(t)$; cf. Remark 3 at the end of $\$ 3$. Methodologically, our paper comes closest to Zacks [22]. In $\S 3$ we demonstrate that this method may also be of considerable value in inventory theory. 
Main contributions of the paper. Our main contributions are the following: (i) We present a detailed performance analysis of a fundamental extension of the classical EOQ model. To the best of our knowledge, the method used to obtain the distribution of the demand process is novel in inventory theory. (ii) We determine the long-run average profit for a natural, given, cost/revenue structure. For this purpose, we determine expressions for the holding costs, outdating costs, and costs for unsatisfied demand per regenerative cycle.

Organization of the paper. Section 2 contains a detailed model description. In $\S \S 3-7$ we analyze the relevant functionals, which are the measures and components of the objective function. Section 3 presents a compound Poisson representation of the problem; Section 4 is devoted to the distribution of an essential stopping time, viz., the minimum of the time till the buffer becomes empty and the time till the buffer content becomes outdated; in $\$ 5$ we consider the residual time from that stopping time until the end of the ongoing low demand period; and in $\S 6$ we consider the amount of discarded material and the average buffer content in a cycle. In $\$ 7$, the special exponential low demand case is discussed. Combining the various results of $\S \S 3-7$ allows us to determine the expected profit per time unit. Numerical examples are given in $\S 8$, in which we explore the effect of several parameters on the cost function.

2. Model formulation and objective function. We consider an inventory model of some fluid, perishable, commodity. The inventory level starts at time $t=0$ at some level $q$. There is a stochastic demand for the commodity. Refilling of the inventory level to level $q$ is required when the first of two events takes place: Either the buffer level reaches zero, or the buffer content becomes outdated (perishes). Below we successively discuss (i) the demand process, (ii) the perishability and replenishment process, and (iii) the cost objective function and optimization problem (determination of the level $q=q^{*}$ that yields the largest net profit per unit of time).

The demand process. The demand for the commodity follows alternating periods of high demand (HD) and low demand (LD). The rate of demand during an HD-period is $\beta_{H}$; during an LD-period it is $\beta_{L}$, where $0<\beta_{L}<\beta_{H}<\infty$. We assume that the lengths of all the successive HD- and LD-periods are independent random variables. The lengths of successive HD-periods are denoted by $\left\{H_{i} ; i=1,2, \ldots\right\}$; they are identically distributed, with distribution $F_{H}(\cdot)$. Throughout the paper, we assume that $F_{H}(t)=1-\mathrm{e}^{-\lambda t}, t \geq 0$. The lengths of successive LD-periods are denoted by $\left\{L_{i} ; i=1,2, \ldots\right\}$; they are identically distributed, with distribution $F_{L}(\cdot)$ and density $f_{L}(\cdot)$. More generally, throughout the paper we denote the distribution and density of a random variable $Z$ by $F_{Z}(\cdot)$ and $f_{Z}(\cdot)$, respectively.

We assume that at time 0 the inventory has just been replenished, and the inventory level is $q$. Let $Q(t)$ denote the amount of commodity demanded in $(0, t)$, with $Q(0):=0$.

The perishability and replenishment process. Define the stopping time

$$
\tau:=\inf \{t>0: Q(t)=q\} .
$$

We define a second key time epoch: Let $t_{0}$, with $0<t_{0}<\infty$, denote the time at which all material in stock is outdated and discarded.

If $\tau \leq t_{0}$, then all material in stock was already demanded before time $t_{0}$, and nothing is discarded.

If $\tau>t_{0}$, then a quantity $q-Q\left(t_{0}\right)$ is discarded. We introduce the amount of discarded material

$$
D(q):=\left(q-Q\left(t_{0}\right)\right) I\left(\tau>t_{0}\right) .
$$

Here $I(\cdot)$ denotes an indicator function. We assume that $q<\beta_{H} t_{0}$; otherwise outdating could never occur.

Define the time

$$
\tau^{*}:=\min \left(\tau, t_{0}\right) .
$$

This is a crucial time epoch in our model. It is the first time after 0 at which the inventory level reaches 0 , either because of the demand or because of outdating. If $\tau^{*}$ occurs in an HD-period, an order is placed that instantaneously brings the inventory level back to $q$. If $\tau^{*}$ occurs in an LD-period, that order is placed at the end of that LD-period. Formally: Replenishment to level $q$ occurs at $\tau^{*}+R$, where $R=0$ if $\tau^{*} \in H D$ whereas, if $\tau^{*} \in L D, R$ is the length of the remainder of the LD-period. Thus, the cycle length, the length of the time between two successive replenishments, is $C=\tau^{*}+R$. To emphasize the fact that $R$ is only positive if $\tau^{*} \in L D$, we write

$$
C=\tau^{*}+R I\left(\tau^{*} \in L D\right) .
$$

In either case, a new cycle starts at the beginning of an HD-period, and with inventory level $q$. The memoryless property of the HD-distribution, combined with the assumption of ordering at the end of an LD-period when $\tau^{*} \in L D$, implies that successive cycles starting with a replenishment probabilistically are indistinguishable. Interreplenishment cycles $C_{1}, C_{2}, \ldots$ form a renewal process, and the inventory level process $\{X(t), t \geq 0\}$, with $X(t)$ the inventory level at time $t$, is regenerative. 
REMARK 2. The above control policy guarantees that each cycle starts with HD rate. The following example shows that it is often natural to wait for the beginning of the next HD period before ordering. Typically during HD-periods trade is relatively brisk. If the holding costs are proportionally high compared to the costs of unsatisfied demand, entrepreneurs will not start new initiatives during LD-periods; if they place orders during these periods the content level will stochastically increase and the holding cost component will cause the long run average costs to rise. If they wait until the beginning of the next HD-period, the lost sales costs are low and the holding costs will also be low, since the content level will be depleted quickly. Hence in this case it may be worthwhile to pay the costs of unsatisfied demands by waiting with the content at level 0 rather than placing an order and for a long time paying considerable holding costs while the content level is near its maximum $q$.

Because of the regenerative structure of the replenishment process, we can restrict ourselves to one cycle $C=C_{1}$. In this cycle, during $\left[0, \tau^{*}\right]$ the demand grows from $Q(0)=0$ as follows:

$$
Q(t)=\beta_{L} t+\left(\beta_{H}-\beta_{L}\right) W(t),
$$

where $W(t)$ is the total length in $(0, t)$ of HD-periods; more formally,

$$
W(t)=\int_{0}^{t} I(s \in H D) \mathrm{d} s,
$$

where $I(s \in H D)$ again is an indicator function. Note that, during $\left[0, \tau^{*}\right]$, the inventory level process and the demand process are related via $X(t)=q-Q(t)$. We need to determine the distribution of $X(t)$ during $\left[0, \tau^{*}\right]$, and hence the distribution of $Q(t)$ during $\left[0, \tau^{*}\right]$, and hence the distribution of $W(t)$ during $\left[0, \tau^{*}\right]$. If $\tau^{*} \in L D$, then the cycle is extended by $R$. Below we take this into account in the cost function as shortage; it is assumed that, during $R$, no orders can be placed, so in particular $Q(t)$ cannot exceed $q$. (One could also make other assumptions, leading to minor adaptations in the model).

The cost objective function and optimization problem. A natural objective is to determine the level $q$ such that the expected net profit per time unit is maximized. Let $s_{l}$ denote the selling price of one unit. Furthermore, let $c_{d}$, $c_{s}$, and $c_{h}$ be the cost of a discarded unit; the costs for shortage of one unit; and the costs of holding the buffer content. Finally, there are set-up costs per cycle; we denote those by $K$. Then the profit objective function, i.e., the net profit per time unit, is

$$
O_{b}(q):=\frac{s_{l} q-K-c_{d} E\{D(q)\}-c_{s} \beta_{L} E\left\{R I\left(\tau^{*} \in L D\right)\right\}-c_{h} E\left\{T\left(\tau^{*}\right)\right\}}{E\{C\}},
$$

where

- $E\{D(q)\}$ denotes the expected amount of discarded commodity,

- $\beta_{L} E\left\{R I\left(\tau^{*} \in L D\right)\right\}$ denotes the expected shortage per cycle (this shortage is only positive when $\tau^{*}$ falls in an LD-period),

- $E\left\{T\left(\tau^{*}\right)\right\}$ denotes the expected quantity of commodity held in stock during $\left(0, \tau^{*}\right)$; here $T\left(\tau^{*}\right):=\int_{0}^{\tau^{*}} X(t) \mathrm{d} t=$ $\int_{0}^{\tau^{*}}(q-Q(t)) \mathrm{d} t$.

Note that, if not all items are not sold, the sales profit in one cycle is less than $s_{l} q$; however, that is taken into account by the $D(q)$ term.

It is clear from (7) that we need to determine the means of $D(q), R I\left(\tau^{*} \in L D\right), T\left(\tau^{*}\right)$ and $C$. In the next two sections we derive the distributions of $W(t), Q(t)$ and $\tau^{*}$ (Theorems 1,2 and 4). That allows us to determine $E\left\{R I\left(\tau^{*} \in L D\right)\right\}$ (Theorem 6) and $E\{C\}=E\left\{\tau^{*}\right\}+E\left\{R I\left(\tau^{*} \in L D\right)\right\}$ (Theorem 7) in $\S 5$ as well as $E\{D\}$ and $E\left\{T\left(\tau^{*}\right)\right\}$ (Theorems 8 and 9) in $\S 6$.

3. Distributions of $W(t)$ and of $Q(t)$. The distribution of $W(t)$ is found in the following manner. Let, for $0<w<\infty$,

$$
N_{H}(w)=\max \left(n \geq 0: \sum_{i=0}^{n} H_{i} \leq w\right)
$$

where $H_{0}=0$ and $H_{i}$ is the length of the $i$-th HD-period. Note that

$$
P\left\{N_{H}(w)=n\right\}=F_{H}^{(n)}(w)-F_{H}^{(n+1)}(w),
$$

where $F_{H}^{(n)}(w)$ denotes the $n$-fold convolution of $F_{H}(w)$. Under the assumption that $H_{i} \sim \exp (\lambda)$, i.e., $F_{H}(w)=$ $1-e^{-\lambda w}, w>0, P\left\{N_{H}(w)=n\right\}=p(n ; \lambda w):=\mathrm{e}^{-\lambda w}(\lambda w)^{n} / n !$, that is the Poisson probability function at $n$, with mean $\lambda w$. Define

$$
Y(w)=\sum_{n=0}^{N_{H}(w)} L_{n},
$$




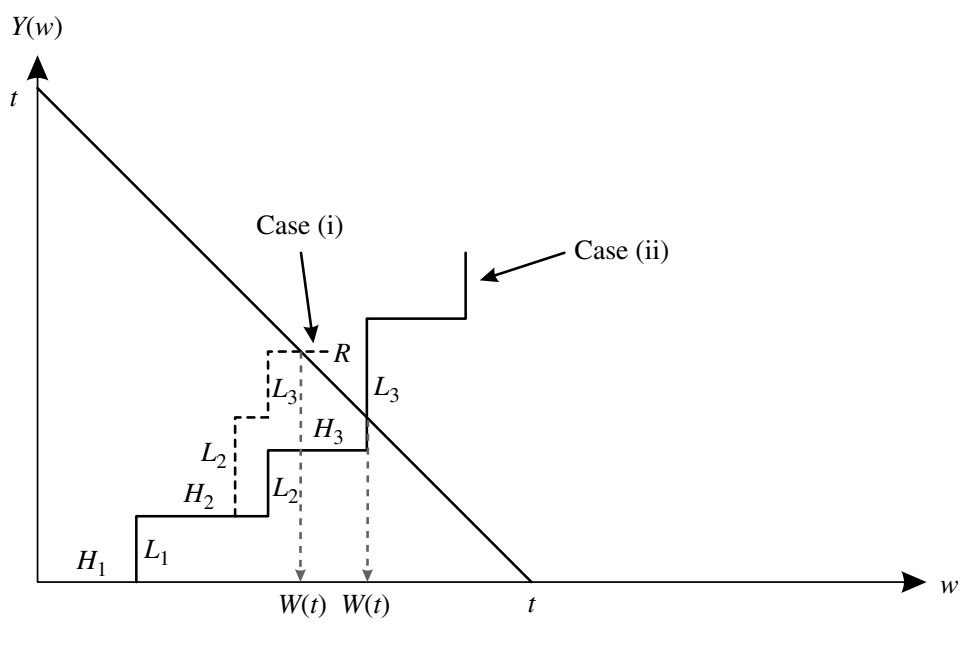

FIGURE 1. Illustration of $W(t)$ and $R$.

where $L_{0}=0$ and $L_{n}$ is the random length of the $n$-th LD-period. Under the exponential assumption for $F_{H}$, $\{Y(w), w \geq 0\}$ is a compound Poisson process. Let $F_{Y}(y ; w)$ denote the c.d.f. of the compound Poisson process $Y(w)$, at $w$. This is given by $F_{Y}(0 ; w)=P\{Y(w)=0\}=e^{-\lambda w}$ (atom of $H$ ), and for $y>0$

$$
F_{Y}(y ; w)=\sum_{n=0}^{\infty} p(n ; \lambda w) F_{L}^{(n)}(y),
$$

where $F_{L}^{(0)}(y) \equiv 1$ and for $n \geq 1, F_{L}^{(n)}(y)$ is the $n$-fold convolution of $F_{L}(y)$. For a given $t, 0<t<\infty, W(t)$ defined in (6) is the stopping time

$$
W(t)=\inf \{w>0: Y(w) \geq t-w\} .
$$

Figure 1 illustrates $W(t)$ and $R$. There are two cases: (i) $t \in$ HD-period; (ii) $t \in$ LD-period. In case (i), the boundary $B(w ; t)=t-w$ is intersected by a horizontal $H$ segment. In this case, if $t=\tau^{*}, R=0$. In case (ii) $Y(W(t))>t-w$ (see vertical segment of $\left.L_{3}\right)$. In this case, if $t=\tau^{*}, R$ is the overshoot $R=Y\left(W\left(\tau^{*}\right)\right)-\left(\tau^{*}-W\left(\tau^{*}\right)\right)$.

THEOREM 1.

$$
P\{W(t)>w\}=F_{Y}(t-w ; w), \quad 0<w<t,
$$

and

$$
P\{W(t)=t\}=e^{-\lambda t} .
$$

Proof. Since $Y(w)$ is an increasing process, as long as $Y(w)<t-w$ there has been no crossing of the boundary $B(w ; t)$, and therefore $W(t)>w$. Finally, $W(t)=t$ if, and only if, $H_{1} \geq t$.

Theorem 2. Let $m(q, t):=\min \left(q, \beta_{H} t\right)$.

$$
P\{Q(t)<x\}=F_{Q}(x ; t)= \begin{cases}0, & \text { if } x \leq \beta_{L} t, \\ 1-F_{Y}\left(\frac{\beta_{H} t-x}{\beta_{H}-\beta_{L}} ; \frac{x-\beta_{L} t}{\beta_{H}-\beta_{L}}\right), & \text { if } \beta_{L} t<x \leq m(q, t), \\ 1, & \text { if } x>m(q, t) .\end{cases}
$$

Also, the distribution has a jump point at $m(q, t)$, namely

$$
P\{Q(t)=m(q, t)\}= \begin{cases}e^{-\lambda t}, & \text { if } t \leq q / \beta_{H}, \\ F_{Y}\left(\frac{\beta_{H} t-q}{\beta_{H}-\beta_{L}} ; \frac{q-t \beta_{L}}{\beta_{H}-\beta_{L}}\right), & \text { if } q / \beta_{H}<t \leq t_{0} .\end{cases}
$$

Proof. Since a cycle starts at an HD-period, $\beta_{L} t<Q(t) \leq \min \left(q, \beta_{H} t\right)=m(q, t)$. The theorem follows from (5) and Theorem 1. Note that the jump $\mathrm{e}^{-\lambda t}$ at $m(q, t)$, for the case $m(q, t)=\beta_{H} t$, is consistent with the second equation of (15): Taking $x=\beta_{H} t$ in that equation gives $P\left\{Q(t)<\beta_{H} t\right\}=1-F_{Y}(0 ; t)=1-\mathrm{e}^{-\lambda t}$.

We now derive the densities of $W(t)$ and of $Q(t)$. Figure 2 presents a sample path of $Q(t)$. 


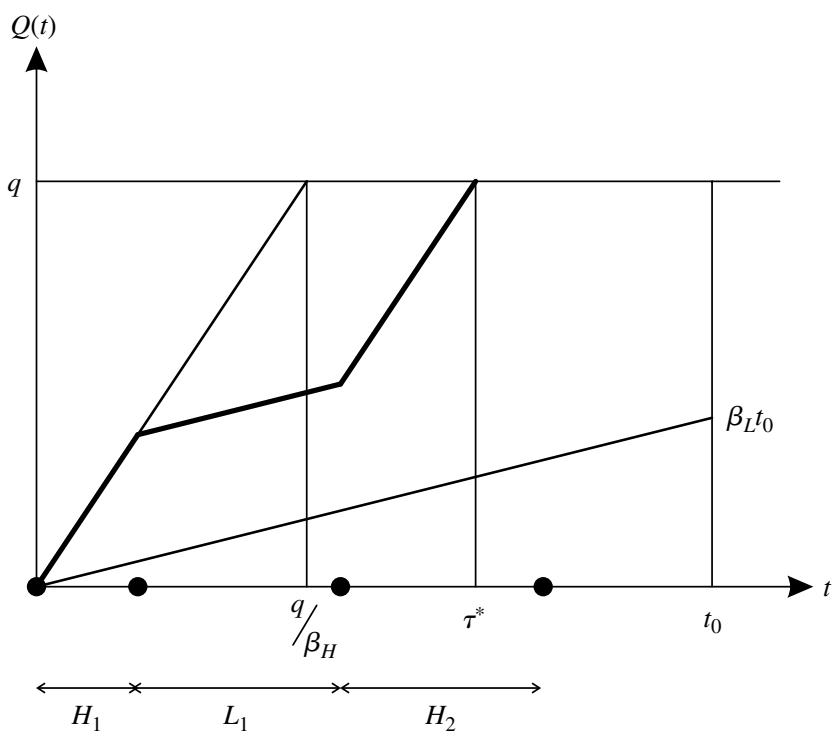

FIGURE 2. $Q(t)$ sample paths. In the present case, $\tau^{*} \in H_{2}$ and therefore $C=\tau^{*}$.

Density of $W(t)$. Let $f_{W(t)}(w)$ denote the density of $W(t)$, for $0<w<t$. From (13) we get

$$
\begin{aligned}
f_{W(t)}(w) & =-\frac{d}{d w} F_{Y}(t-w ; w)=-\frac{d}{d w} \sum_{n=0}^{\infty} p(n ; \lambda w) F_{L}^{(n)}(t-w) \\
& =\lambda \sum_{n=0}^{\infty}(p(n ; \lambda w)-p(n-1 ; \lambda w)) F_{L}^{(n)}(t-w)+\sum_{n=1}^{\infty} p(n ; \lambda w) f_{L}^{(n)}(t-w),
\end{aligned}
$$

where $p(-1 ; \lambda w) \equiv 0$ and $f_{L}^{(n)}(\cdot)$ is the $n$-fold convolution of the density $f_{L}(\cdot)$ of $L$. Note that the last term on the right-hand side of (17) is the density of $Y(w)$ at $t-w$, i.e., $f_{Y}(t-w ; w)$, where for $y>0$

$$
f_{Y}(y ; w)=\sum_{n=1}^{\infty} p(n ; \lambda w) f_{L}^{(n)}(y) .
$$

Furthermore, since

$$
F_{Y}(y ; w)=P(Y(w) \leq y)=P(Y(w) \leq y, w \in \mathrm{LD})+P(Y(w) \leq y, w \in \mathrm{HD}),
$$

then as shown in Figure 1, in case (i) the sample path of $Y(w)$ is equal to $t-w$ when $W(t)=w$. Thus, in this case,

$$
f_{Y}(t-w ; w)=\frac{d}{d w} P(Y(w) \leq t-w, w \in \mathrm{HD}) .
$$

We therefore denote the first of the two rightmost terms of $(17)$ by $f_{W(t)}^{(\mathrm{LD})}(w)$ and the second term by $f_{W(t)}^{(\mathrm{HD})}(w)$. Since $f_{W(t)}(w)=f_{W(t)}^{(\mathrm{LD})}(w)+f_{W(t)}^{(\mathrm{HD})}(w)$, we also obtain that

$$
P(t \in \mathrm{HD} \mid W(t)=w)=\frac{f_{W(t)}^{(\mathrm{HD})}(w)}{f_{W(t)}(w)} .
$$

Density of $Q(t)$. Differentiating $F_{Q}(x ; t)$ as given in (15) with respect to $x$, we obtain the density of $Q(t)$ on $\left(\beta_{L} t, \min \left(q, \beta_{H} t\right)\right)$, as follows:

$$
\begin{aligned}
f_{Q}(x ; t)= & \frac{\lambda}{\Delta}\left[e^{-\lambda\left(x-\beta_{L} t\right) / \Delta}+\sum_{n=1}^{\infty}\left(p\left(n ; \lambda \frac{x-\beta_{L} t}{\Delta}\right)-p\left(n-1 ; \lambda \frac{x-\beta_{L} t}{\Delta}\right)\right) F_{L}^{(n)}\left(\frac{\beta_{H} t-x}{\Delta}\right)\right] \\
& +\frac{1}{\Delta} \sum_{n=1}^{\infty} p\left(n ; \lambda \frac{x-\beta_{L} t}{\Delta}\right) f_{L}^{(n)}\left(\frac{\beta_{H} t-x}{\Delta}\right),
\end{aligned}
$$

where $\Delta=\beta_{H}-\beta_{L}$. 
Finally, we give expressions for the mean of $W(t)$ and $Q(t)$.

THEOREM 3.

$$
E\{W(t)\}=\int_{0}^{t} F_{Y}(t-y ; y) d y, \quad 0<t \leq t_{0},
$$

and

$$
\begin{aligned}
E\{Q(t)\}= & \beta_{L} t+\Delta E\left\{W(t) I\left(\beta_{L} t<Q(t) \leq m(q, t)\right)\right\} \\
& = \begin{cases}\beta_{L} t+\Delta E\{W(t)\}, & \text { if } t \leq \frac{q}{\beta_{H}}, \\
\beta_{L} t+\Delta \int_{0}^{\left(q-\beta_{L} t\right) / \Delta} F_{Y}(t-y ; y) \mathrm{d} y, & \text { if } t>\frac{q}{\beta_{H}} .\end{cases}
\end{aligned}
$$

Proof. Equation (21) immediately follows from

$$
E\{W(t)\}=\int_{0}^{\infty} P(W(t)>y) \mathrm{d} y=\int_{0}^{t} F_{Y}(t-y, y) \mathrm{d} y .
$$

To prove (22), distinguish between $t \leq q / \beta_{H}$ and $t>q / \beta_{H}$. If $t \leq q / \beta_{H}$, then $\beta_{L} t-\Delta W(t) \leq \beta_{H} t$, or equivalently, $0<W(t) \leq t\left(\left(\beta_{H}-\beta_{L}\right) / \Delta\right)=t$. Thus, if $t \leq q / \beta_{H}$ then $E\{W(t) I(0<W(t) \leq t)\}=E\{W(t)\}$ as in (21). On the other hand, if $t>q / \beta_{H}$ then $\{Q(t) \leq q\}$ or $\left\{0<W(t) \leq\left(q-\beta_{L} t\right) / \Delta\right\}$. Also, since $\beta_{H} t>q$ when $t>q / \beta_{H}$,

$$
E\{Q(t)\}=q F_{Y}\left(\frac{\beta_{H} t-q}{\Delta} ; \frac{q-\beta_{L} t}{\Delta}\right)+\int_{\beta_{L} t}^{q} x f_{Q}(x ; t) \mathrm{d} x .
$$

Still

$$
\begin{aligned}
\int_{\beta_{L^{t}}}^{q} x f_{Q}(x ; t) \mathrm{d} x & =q F_{Q}(q ; t)-\int_{\beta_{L} t}^{q} F_{Q}(y ; t) \mathrm{d} y \\
& =q\left(1-F_{Y}\left(\frac{\beta_{H} t-q}{\Delta} ; \frac{q-\beta_{L} t}{\Delta}\right)\right)-\int_{\beta_{L} t}^{q}\left(1-F_{Y}\left(\frac{\beta_{H} t-y}{\Delta} ; \frac{y-\beta_{L} t}{\Delta}\right)\right) \mathrm{d} y .
\end{aligned}
$$

Hence

$$
E\{Q(t)\}=\beta_{L} t+\Delta \int_{0}^{\left(q-\beta_{L} t\right) / \Delta} F_{Y}(t-y ; y) \mathrm{d} y .
$$

This proves (22). Note that $t_{0}<q / \beta_{L}$.

Remark 3. The distribution of the inventory level, $X(t)$, which equals $q-Q(t)$ for $t \in\left[0, \tau^{*}\right]$, can easily be derived from that of $Q(t)$. Indeed, $P(X(t) \leq x)=P(Q(t) \geq q-x)$ for $t \leq \tau^{*}, 0 \leq x \leq q$. Furthermore, $X(t) \equiv 0$ for $t \in\left(\tau^{*}, C\right]$ : either $Q\left(\tau^{*}\right)=q$, hence $X\left(\tau^{*}\right)=0$, or $\tau^{*}=t_{0}$ and the remaining inventory is discarded.

4. The distribution of $\tau^{*}$. This section is devoted to the stopping time $\tau^{*}$, the minimum time until the buffer level reaches 0 in a cycle and the buffer content becomes outdated. Recall first that $q / \beta_{H} \leq \tau^{*} \leq t_{0}$. Thus we get

THEOREM 4. For $q / \beta_{H} \leq t<t_{0}$,

$$
P\left\{\tau^{*} \leq t\right\}=F_{Y}\left(\frac{\beta_{H} t-q}{\Delta} ; \frac{q-\beta_{L} t}{\Delta}\right)
$$

and

$$
P\left\{\tau^{*}=t_{0}\right\}=1-F_{Y}\left(\frac{\beta_{H} t_{0}-q}{\Delta} ; \frac{q-\beta_{L} t_{0}}{\Delta}\right) .
$$

Proof. We have $P\left\{\tau^{*}>t\right\}=P\{Q(t) \leq q\}$. Thus, we obtain (25) from (15). Also, $P\left\{\tau^{*}=t_{0}\right\}=P\left\{\tau^{*} \geq t_{0}\right\}$ since $\tau^{*} \leq t_{0}$. This yields (26).

Note that (25) implies that $P\left\{\tau^{*}=q / \beta_{H}\right\}=e^{-\lambda q / \beta_{H}}$. The density of $\tau^{*}$ on $\left(q / \beta_{H}, t_{0}\right)$ is obtained by differentiating (25) with respect to $t$. We get $f_{\tau^{*}}(t ; q)=f_{\tau^{*}}^{(\mathrm{LD})}(t ; q)+f_{\tau^{*}}^{(\mathrm{HD})}(t ; q)$, where

$$
f_{\tau^{*}}^{(\mathrm{LD})}(t ; q)=\lambda \frac{\beta_{L}}{\Delta}\left[e^{-\lambda\left(q-\beta_{L} t\right) / \Delta}+\sum_{n=1}^{\infty}\left(p\left(n ; \lambda \frac{q-\beta_{L} t}{\Delta}\right)-p\left(n-1 ; \lambda \frac{q-\beta_{L} t}{\Delta}\right)\right) F_{L}^{(n)}\left(\frac{\beta_{H} t-q}{\Delta}\right)\right]
$$


and

$$
f_{\tau^{*}}^{(\mathrm{HD})}(t ; q)=\frac{\beta_{H}}{\Delta} \sum_{n=1}^{\infty} p\left(n ; \lambda \frac{q-\beta_{L} t}{\Delta}\right) f_{L}^{(n)}\left(\frac{\beta_{H} t-q}{\Delta}\right) .
$$

As before, $f_{\tau^{*}}^{(\mathrm{LD})}(t ; q)=(d / d t) P\left(\tau^{*} \leq t, \tau^{*} \in \mathrm{LD}\right)$ and $f_{\tau^{*}}^{(\mathrm{HD})}(t ; q)=(d / d t) P\left(\tau^{*} \leq t, \tau^{*} \in \mathrm{HD}\right)$. Similar to (19),

$$
P\left\{\tau^{*} \in \mathrm{HD} \mid \tau^{*}=t\right\}=\frac{f_{\tau^{*}}^{(\mathrm{HD})}(t ; q)}{f_{\tau^{*}}(t ; q)} .
$$

Finally we prove,

THEOREM 5. The expected value of $\tau^{*}$ is

$$
E\left\{\tau^{*}\right\}=t_{0}-\int_{q / \beta_{H}}^{t_{0}} F_{Y}\left(\frac{\beta_{H} x-q}{\Delta} ; \frac{q-\beta_{L} x}{\Delta}\right) \mathrm{d} x .
$$

Proof. Using that $P\left\{\tau^{*}>t\right\}=1$ for all $t<q / \beta_{H}$, we can write:

$$
\begin{aligned}
E\left\{\tau^{*}\right\} & =\int_{0}^{\infty} P\left\{\tau^{*}>t\right\} \mathrm{d} t=\frac{q}{\beta_{H}}+\int_{q / \beta_{H}}^{t_{0}}\left(1-F_{Y}\left(\frac{\beta_{H} t-q}{\Delta} ; \frac{q-\beta_{L} t}{\Delta}\right)\right) \mathrm{d} t \\
& =t_{0}-\int_{q / \beta_{H}}^{t_{0}} F_{Y}\left(\frac{\beta_{H} t-q}{\Delta} ; \frac{q-\beta_{L} t}{\Delta}\right) \mathrm{d} t .
\end{aligned}
$$

5. The distribution of $R$. As mentioned in $\S 3$, the overshoot at stopping is

$$
\begin{aligned}
R & =W\left(\tau^{*}\right)+Y\left(W\left(\tau^{*}\right)\right)-\tau^{*} \\
& = \begin{cases}0, & \text { if } \tau^{*} \in \mathrm{HD}, \\
>0, & \text { if } \tau^{*} \in \mathrm{LD} .\end{cases}
\end{aligned}
$$

Accordingly, $E\{R\}=E\left\{R I\left(\tau^{*} \in L D\right)\right\}$. Our goal in this section is to determine this expectation.

Generally, if $Y(t)=\sum_{n=1}^{N(t)} X_{n}$ is a compound Poisson process with Poisson arrival rate $\lambda$ and density $f_{X}(\cdot)$ of the $X_{n}$, and $B(t)$ is a nondecreasing linear boundary, then the joint density of the first crossing time, $\theta$, and the overshoot $R=Y(\theta)-B(\theta)$ is (see Zacks [23], Stadje and Zacks [19]):

$$
f_{\theta, R}(t, r)=\lambda \mathrm{e}^{-\lambda t} f_{X}(B(t)+r)+\lambda \int_{0}^{B(t)} f_{X}(B(t)+r-y) \mathrm{d}_{y} P(Y(t) \leq y, \theta>t) .
$$

In the present study, the boundary is $B(w ; t)=t-w$, for $0 \leq w \leq t$ (see Figure 1). Thus, in this case $\mathrm{d}_{y} P(Y(t) \leq y, \tau>t)=f_{Y}(y ; t) \mathrm{d} y$. Taking into account that in the present study $\theta=W(t), X_{n}=L_{n}$ and that $R$ has an atom at 0 , which equals $P\left(\tau^{*} \in H D\right)$, we find:

$$
\begin{aligned}
f_{W(t), R}\left(w, r \mid \tau^{*}=t, \tau^{*} \in \mathrm{LD}\right)= & \lambda e^{-\lambda w} f_{L}(t-w+r) \\
& +\lambda \int_{0}^{t-w} f_{Y}(x ; w) f_{L}(t-w-x+r) \mathrm{d} x .
\end{aligned}
$$

Note that if $f_{L}(x)=\mu e^{-\mu x}, x>0$, then

$$
f_{W(t), R}\left(w, r \mid \tau^{*}=t, \tau^{*} \in \mathrm{LD}\right)=\mu e^{-\mu r}\left[\lambda e^{-\lambda w-\mu(t-w)}+\lambda \int_{0}^{t-w} f_{Y}(x ; w) e^{-\mu(t-w-x)} \mathrm{d} x\right] .
$$

Thus, in the exponential case, $R$ is independent of $\left(W(t), \tau^{*} \in \mathrm{LD}, \tau^{*}=t\right)$.

The marginal conditional density of $W(t)$ is

$$
f_{W(t)}\left(w \mid \tau^{*}=t, \tau^{*} \in \mathrm{LD}\right)=\lambda e^{-\lambda w} \bar{F}_{L}(t-w)+\lambda \int_{0}^{t-w} f_{Y}(x ; w) \bar{F}_{L}(t-w-x) \mathrm{d} x .
$$

Finally, since $\int_{0}^{\infty} r f_{L}(\alpha+r) \mathrm{d} r=\int_{\alpha}^{\infty} \bar{F}_{L}(u) \mathrm{d} u$, we obtain from (34):

$$
E\left\{R \mid \tau^{*}=t, \tau^{*} \in \mathrm{LD}, W(t)=w\right\}=\frac{\lambda e^{-\lambda w} \int_{t-w}^{\infty} \bar{F}_{L}(u) \mathrm{d} u+\lambda \int_{0}^{t-w} f_{Y}(x ; w) \int_{t-w-x}^{\infty} \bar{F}_{L}(u) \mathrm{d} u \mathrm{~d} x}{f_{W(t)}\left(w \mid \tau^{*}=t, \tau^{*} \in \mathrm{LD}\right)}
$$


It is easily verified from (37) that if $L \sim \operatorname{Exp}(\mu)$, then $E\left\{R \mid \tau^{*}=t, \tau^{*} \in \mathrm{LD}, W(t)=w\right\}=1 / \mu=E\{R\}$ (conditioned to be positive). Indeed, in the exponential case $R$ is independent of $\tau^{*}$ and of $W\left(\tau^{*}\right)$.

In the general case, if $q / \beta_{H}<t<t_{0}$, then

$$
E\left\{R \mid \tau^{*}=t, \tau^{*} \in \mathrm{LD}\right\}=\lambda \int_{0}^{t} e^{-\lambda w} \int_{t-w}^{\infty} \bar{F}_{L}(u) \mathrm{d} u \mathrm{~d} w+\lambda \int_{0}^{t} \int_{0}^{t-w} f_{Y}(x ; w) \int_{t-w-x}^{\infty} \bar{F}_{L}(u) \mathrm{d} u \mathrm{~d} x \mathrm{~d} w .
$$

When $t=t_{0}$,

$$
E\left\{R \mid \tau^{*}=t_{0}, t_{0} \in L D\right\}=\frac{\int_{0}^{\left(q-t_{0} \beta_{L}\right) / \Delta} f_{Q}\left(\beta_{L} t_{0}+\Delta w ; t_{0}\right) f_{W\left(t_{0}\right)}^{(L D)}(w) E\left\{R \mid \tau^{*}=t_{0}, t_{0} \in L D, W\left(t_{0}\right)=w\right\} \mathrm{d} w}{\int_{0}^{\left(q-t_{0} \beta_{L}\right) / \Delta} f_{Q}\left(\beta_{L} t_{0}+\Delta w ; t_{0}\right) f_{W\left(t_{0}\right)}^{(L D)}(w) \mathrm{d} w} .
$$

Finally, from (38) and (39) we get:

THEOREM 6.

$$
\begin{aligned}
E\left\{R I\left(\tau^{*} \in L D\right)\right\}= & \int_{q / \beta_{H}}^{t_{0}} f_{\tau^{*}}^{(L D)}(t ; q) E\left\{R \mid \tau^{*}=t, t \in L D\right\} \mathrm{d} t \\
& +\int_{0}^{\left(q-\beta_{L} t_{0}\right) / \Delta} f_{Q}\left(\beta_{L} t_{0}+\Delta w ; t_{0}\right) f_{W\left(t_{0}\right)}^{(L D)}(w) E\left\{R \mid \tau^{*}=t_{0}, t_{0} \in L D, W\left(t_{0}\right)=w\right\} \mathrm{d} w .
\end{aligned}
$$

Combining (4), Theorem 5 and Theorem 6 gives the mean cycle time:

THEOREM 7.

$$
\begin{aligned}
E\{C\}= & E\left\{\tau^{*}\right\}+E\left\{R I\left(\tau^{*} \in L D\right)\right\} \\
= & t_{0}-\int_{q / \beta_{H}}^{t_{0}} F_{Y}\left(\frac{\beta_{H} x-q}{\Delta} ; \frac{q-\beta_{L} x}{\Delta}\right) \mathrm{d} x+\int_{q / \beta_{H}}^{t_{0}} f_{\tau^{*}}^{(L D)}(t ; q) E\left\{R \mid \tau^{*}=t, t \in L D\right\} \mathrm{d} t \\
& +\int_{0}^{\left(q-\beta_{L} t_{0}\right) / \Delta} f_{Q}\left(\beta_{L} t_{0}+\Delta w ; t_{0}\right) f_{W\left(t_{0}\right)}^{(L D)}(w) E\left\{R \mid \tau^{*}=t_{0}, t_{0} \in L D, W\left(t_{0}\right)=w\right\} \mathrm{d} w .
\end{aligned}
$$

6. Discarded material and total holding quantities. This section contains two theorems. Theorem 8 presents the mean amount of discarded material per cycle, and Theorem 9 the mean total holding quantity per cycle.

THEOREM 8. The expected amount of discarded material is

$$
E\{D(q)\}=q-\beta_{L} t_{0}-\Delta \int_{0}^{\left(q-\beta_{L} t_{0}\right) / \Delta} F_{Y}\left(t_{0}-y ; y\right) d y .
$$

Proof. Use $D(q)=\left(q-Q\left(t_{0}\right)\right) I\left(\tau>t_{0}\right)=q-Q\left(t_{0}\right)$ in combination with (22) (note that $\left.t_{0}>q / \beta_{H}\right)$.

THEOREM 9. The expected total holding quantity per cycle is

$$
\begin{aligned}
E\left\{T\left(\tau^{*}\right)\right\}= & E\left\{\int_{0}^{\tau^{*}}(q-Q(t)) \mathrm{d} t\right\} \\
= & \frac{q^{2}}{2 \beta_{H}} e^{-\lambda q / \beta_{H}}+\int_{q / \beta_{H}}^{t_{0}} f_{\tau^{*}}(t ; q) \int_{0}^{t} E\left\{q-Q(s) \mid \tau^{*}=t\right\} \mathrm{d} s \mathrm{~d} t \\
& +\int_{\beta_{L} t_{0}}^{q} f_{Q}\left(y ; t_{0}\right)\left[q-\int_{0}^{t_{0}} E\left\{Q(s) \mid Q\left(t_{0}\right)=y\right\} \mathrm{d} s\right] \mathrm{d} y .
\end{aligned}
$$

Here $f_{\tau^{*}}(t ; q)$ is obtained by adding the expressions in (27) and (28). The density of $f_{Q}(x ; t)$ is given by (20). $E\left\{Q(s) \mid \tau^{*}=t\right\}$ is given in Lemma 1 below, and $E\left\{Q(s) \mid Q\left(t_{0}\right)=y\right\}$ is given in Lemma 2 below.

Proof. The first equality follows by definition. The second equality is straightforward.

What remains is to determine $E\left\{Q(s) \mid Q\left(t_{0}\right)=y\right\}$ and $E\left\{Q(s) \mid \tau^{*}=t\right\}$, where $0<s<t$, and $q / \beta_{H}<t<t_{0}$. Note that $\left\{\tau^{*}=t\right\}=\left\{Q(u)<q\right.$, for all $q / \beta_{H}<u<t$, and $\left.Q(t)=q\right\}$. In Figure 3 below we present the domain, $D$, of all sample paths, which lead to $\left\{\tau^{*}=t\right\}$. Note that for all these sample paths $W(t)=\left(q-\beta_{L} t\right) / \Delta$. The figure shows two possible sample paths, for which $Q(s)=y$. 


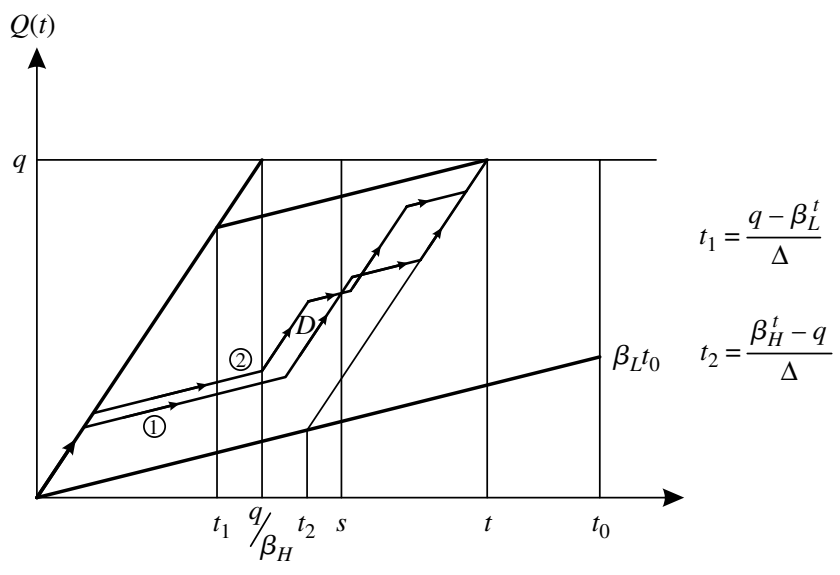

FIGURE 3. Domain of sample paths for $\left\{\tau^{*}=t\right\}$.

Sample path (1) crosses the level $Q(s)=y$ while $s \in$ HD period. Sample path (2) crosses $Q(s)=y$ while $s \in \mathrm{LD}$ period. For both these sample paths $W(s)=\left(y-\beta_{L} s\right) / \Delta$. Let $f_{W(s), W(t)}(z, w)$ denote the joint density of $(W(s), W(t)), 0<s<t$. This joint density can be written as the sum of $f_{W(s), W(t)}^{(\mathrm{HD})}(z, w)$ and $f_{W(s), W(t)}^{(\mathrm{LD})}(z, w)$. Because of the strong Markov property of the compound Poisson process $Y(t)$, for any $0<s<t, z<s$,

$$
f_{W(s), W(t)}^{(\mathrm{HD})}(z, w)=f_{W(s)}^{(\mathrm{HD})}(z) f_{W(t-s)}(w-z) .
$$

On the other hand, when $s \in \mathrm{LD}$,

$$
f_{W(s), W(t)}^{(\mathrm{LD})}(z, w)=\int_{0}^{t-w-(s-z)} f_{W(s), R_{W(s)}^{(\mathrm{LD}}}(z, r) f_{W(t-s-r)}(w-z) \mathrm{d} r .
$$

Thus, with $f_{W(s), W(t)}(z, w)=f_{W(s), W(t)}^{(\mathrm{HD})}(z, w)+f_{W(s), W(t)}^{(\mathrm{LD})}(z, w)$, the joint density of $(Q(s), Q(t))$ is

$$
f_{Q(s), Q(t)}(y, q)=\frac{1}{\Delta^{2}} f_{W(s), W(t)}\left(\frac{y-\beta_{L} s}{\Delta}, \frac{q-\beta_{L} t}{\Delta}\right) .
$$

Finally, define the limits (cf. the definition of $t_{1}$ and $t_{2}$ in Figure 3 above):

$$
\xi_{L}(s)= \begin{cases}\beta_{L} s, & 0<s \leq t_{2} \\ \beta_{L} t_{2}+\beta_{H}\left(s-t_{2}\right), & t_{2}<s<t\end{cases}
$$

and

$$
\xi_{U}(s)= \begin{cases}\beta_{H} s, & s \leq t_{1} \\ \beta_{H} t_{1}+\beta_{L}\left(s-t_{1}\right), & t_{1}<s<t .\end{cases}
$$

Then,

Lemma 1. For every $0<s<t$,

$$
E\left\{Q(s) \mid \tau^{*}=t\right\}=\frac{\int_{\xi_{L}(s)}^{\xi_{U}(s)} y f_{Q(s), Q(t)}(y, q) \mathrm{d} y}{\int_{\xi_{L}(s)}^{\xi_{U}(s)} f_{Q(s), Q(t)}(y, q) \mathrm{d} y} .
$$

Define the limits

$$
\xi_{L}(s, y)= \begin{cases}\beta_{L} s, & 0<s \leq t_{2}(y) \\ \beta_{L} t_{2}(y)+\beta_{H}\left(s-t_{2}(y)\right), & t_{2}(y)<s<t\end{cases}
$$

and

$$
\xi_{U}(s)= \begin{cases}\beta_{H} s, & s \leq t_{1}(y) \\ \beta_{H} t_{1}(y)+\beta_{L}\left(s-t_{1}(y)\right), & t_{1}(y)<s<t,\end{cases}
$$

with $t_{1}(y):=\left(y-\beta_{L} t_{0}\right) / \Delta$ and $t_{2}(y):=\left(\beta_{H} t_{0}-y\right) / \Delta$. 
LeMma 2. For $\beta_{L} t_{0}<y<q$,

$$
E\left\{Q(s) \mid Q\left(t_{0}\right)=y\right\}=\frac{\int_{\xi_{L}(s, y)}^{\xi_{U}(s, y)} x f_{Q(s), Q\left(t_{0}\right)}(x, y) \mathrm{d} x}{\int_{\xi_{L}(s, y)}^{\xi_{U}(s, y)} f_{Q(s), Q\left(t_{0}\right)}(x, y) \mathrm{d} x} .
$$

Proof. Similar to the proof of Lemma 1.

7. Exponential functionals. In this section we develop the equations of the previous sections for the case wherein $F_{L}(y)=1-e^{-\mu y}, y \geq 0$. In this case, according to the Gamma-Poisson relationship,

$$
\begin{aligned}
F_{L}^{(n)}(y) & =P\{G(n ; \mu) \leq y\} \\
& =1-P(n-1 ; \mu y),
\end{aligned}
$$

where $P(j ; x)=\sum_{i=0}^{j} p(i ; x)$ is the c.d.f. of a Poisson distribution with mean $x$ and $G(n, \mu)$ is the Erlang $(n, \mu)$ random variable. Thus, (11) becomes

$$
\begin{aligned}
F_{Y}(y ; w) & =\sum_{n=0}^{\infty} p(n ; \lambda w)[1-P(n-1 ; \mu y)] \\
& =\sum_{j=0}^{\infty} p(j ; \mu y) P(j ; \lambda w) .
\end{aligned}
$$

The density $f_{Y}(y ; w)$ of $F_{Y}(y ; w)$, for $y>0$, is

$$
f_{Y}(y ; w)=\mu \sum_{n=1}^{\infty} p(n ; \lambda w) p(n-1 ; \mu y) .
$$

Accordingly, by (13) and (14),

$$
P\{W(t)>w\}=\sum_{j=0}^{\infty} p(j ; \mu(t-w)) P(j ; \lambda w)
$$

and

$$
P\{W(t)=t\}=e^{-\lambda t}
$$

Following (15), the c.d.f. of $Q(t)$ is

$$
F_{Q}(x ; t)= \begin{cases}0, & \text { if } x \leq \beta_{L} t, \\ 1-\sum_{j=0}^{\infty} p\left(j ; \mu \frac{\beta_{H} t-x}{\Delta}\right) P\left(j ; \lambda \frac{x-\beta_{L} t}{\Delta}\right), & \text { if } \beta_{L} t<x<m(q, t), \\ 1, & \text { if } x \geq m(q, t) .\end{cases}
$$

Also, from (16),

$$
P\{Q(t)=m(q, t)\}= \begin{cases}e^{-\lambda t}, & \text { if } t \leq q / \beta_{H}, \\ \sum_{j=0}^{\infty} p\left(j ; \mu \frac{\beta_{H} t-q}{\Delta}\right) P\left(j ; \lambda \frac{q-\beta_{L} t}{\Delta}\right), & \text { if } q / \beta_{H}<t \leq t_{0} .\end{cases}
$$

After some manipulations we obtain that the LD- and HD-densities of $W(t)$ are

and

$$
f_{W(t)}^{(\mathrm{LD})}(w)=\lambda \sum_{n=0}^{\infty} p(n ; \lambda w) p(n ; \mu(t-w))
$$

$$
f_{W(t)}^{(\mathrm{HD})}(w)=\mu \sum_{n=1}^{\infty} p(n ; \lambda t) p(n-1 ; \mu(t-w)) .
$$


Boxma, Perry, and Zacks: Fluid EOQ of Perishable Items

TABLE 1. $E\left\{Q(s) \mid \tau^{*}=t\right\}$ for $q=300$.

\begin{tabular}{rrrr}
\hline $\mathrm{s} / \mathrm{t}$ & 10 & 15 & \multicolumn{1}{c}{20} \\
\hline 2 & 60 & 40.66 & 39.94 \\
4 & 120 & 82.32 & 79.03 \\
6 & 180 & 124.91 & 108.22 \\
8 & 240 & 160.35 & 129.14 \\
10 & 300 & 192.78 & 150.54 \\
12 & & 227.30 & 172.89 \\
14 & & 262.64 & 197.71 \\
16 & 300.00 & 229.73 \\
18 & & & 263.85 \\
20 & & & 300.00 \\
\hline
\end{tabular}

TABLE 2. Several functionals

\begin{tabular}{lccrr}
\hline$q$ & $E\left\{\tau^{*}\right\}$ & $E\left\{R I\left\{\tau^{*} \in \mathrm{LD}\right\}\right\}$ & $E\{D(q)\}$ & $E\left\{T\left(\tau^{*}\right)\right\}$ \\
\hline 250 & 10.307 & 3.5501 & 0.0023 & $1,268.743$ \\
300 & 12.438 & 3.4770 & 0.1519 & $1,832.027$ \\
350 & 14.535 & 3.3264 & 0.8558 & $2,477.871$ \\
400 & 16.564 & 3.0686 & 2.8427 & $3,167.589$ \\
450 & 18.467 & 2.6857 & 7.2678 & $3,852.358$ \\
500 & 20.180 & 2.1828 & 15.6576 & $4,443.355$ \\
\hline
\end{tabular}

Similarly, we get from (20) that

$$
f_{Q}(x ; t)=\frac{\lambda}{\Delta} \sum_{n=0}^{\infty} p\left(n ; \lambda \frac{x-\beta_{L} t}{\Delta}\right) p\left(n ; \mu \frac{\beta_{H} t-x}{\Delta}\right) .
$$

According to Equations (15), (25) and (58)

$$
P\left\{\tau^{*} \leq t\right\}=\sum_{j=0}^{\infty} p\left(j ; \mu \frac{\beta_{H} t-q}{\Delta}\right) P\left(j ; \lambda \frac{q-\beta_{L} t}{\Delta}\right),
$$

and, according to (26),

$$
P\left\{\tau^{*}=t_{0}\right\}=1-\sum_{j=0}^{\infty} p\left(j ; \mu \frac{\beta_{H} t_{0}-q}{\Delta}\right) P\left(j ; \lambda \frac{q-\beta_{l} t_{0}}{\Delta}\right) .
$$

Several manipulations reduce Equation (27) to

$$
f_{\tau^{*}}^{(\mathrm{LD})}(t ; q)=\lambda \frac{\beta_{L}}{\Delta} \sum_{n=0}^{\infty} p\left(n ; \mu \frac{\beta_{H} t-q}{\Delta}\right) p\left(n ; \lambda \frac{q-\beta_{L} t}{\Delta}\right),
$$

and

$$
f_{\tau^{*}}^{(\mathrm{HD})}(t ; q)=\mu \frac{\beta_{H}}{\Delta} \sum_{n=1}^{\infty} p\left(n ; \lambda \frac{q-\beta_{L} t}{\Delta}\right) p\left(n-1 ; \mu \frac{\beta_{H} t-q}{\Delta}\right) .
$$

TABLE 3. Values of $O_{b}(q)$.

\begin{tabular}{lcccccc}
\hline$q$ & $c_{h}=0$ & $c_{h}=0.05$ & $c_{h}=0.10$ & $c_{h}=0.15$ & $c_{h}=0.20$ & $c_{h}=0.21$ \\
\hline 250 & 87.947 & 83.368 & 78.791 & 74.213 & 69.635 & 68.719 \\
300 & 92.254 & 86.499 & 80.742 & 74.987 & 69.231 & 68.080 \\
350 & 96.012 & 89.076 & 82.139 & 75.203 & 68.266 & 66.879 \\
400 & 99.657 & 91.581 & 83.506 & 75.431 & 67.353 & 65.740 \\
450 & 103.073 & 93.967 & 84.861 & 75.755 & 66.649 & 64.828 \\
500 & 106.539 & 96.624 & 86.690 & 76.755 & 66.820 & 64.833 \\
\hline
\end{tabular}


In the present exponential case, Equation (40) is given by

$$
\begin{aligned}
E\left\{R I\left(\tau^{*} \in \mathrm{LD}\right)\right\} & =\frac{1}{\mu} P\left\{\tau^{*} \in L D\right\} \\
& =\frac{1}{\mu}\left[\int_{q / \beta_{H}}^{t_{0}} f_{\tau^{*}}^{(\mathrm{LD})}(t ; q) \mathrm{d} t+\int_{0}^{\left(q-\beta_{L} t_{0}\right) / \Delta} f_{Q}\left(\beta_{L} t_{0}+\Delta w ; t_{0}\right) f_{W\left(t_{0}\right)}^{(L D)}(w) \mathrm{d} w\right] .
\end{aligned}
$$

8. Some numerical results. In this section we present numerical results for some of the key performance measures, for the case of exponential LD periods. In all the tables the following parameters were used: $\lambda=0.1$, $\mu=0.2, \beta_{H}=30, \beta_{L}=10$ and $t_{0}=24$. Other parameters, such as $q$, were varied.

In Table 1, we present $E\left\{Q(s) \mid \tau^{*}=t\right\}$ for $q=300$, and for various values of $s$ and $t$.

In Table 2 we present the functionals $E\left\{\tau^{*}\right\}, E\left\{R I\left(\tau^{*} \in L D\right)\right\}, E\left\{D\left(Q ; t_{0}\right)\right\}$ and $E\left\{T\left(\tau^{*}\right)\right\}$ for several values of $q$. Note that $E\left\{T\left(\tau^{*}\right)\right\}$ should belong to the interval $\left(q^{2} /\left(2 \beta_{H}\right), q t_{0}-\beta_{L} t_{0}^{2} / 2\right)$.

Finally we present in Table 3 a few values of the expected profit objective function $O_{b}(q)$, cf. (7), which correspond to the values in Table 2. We state that $E\{C\}=E\left\{\tau^{*}\right\}+E\left\{R I\left(\tau^{*} \in L D\right)\right\}$. In this table we have used the cost values $s_{l}=5, K=10, c_{s}=6.0, c_{d}=6.0$ and various values of $c_{h}$.

According to the values in Table 3 , if $c_{h}<0.20$ it is optimal to use $q=500$ (largest). On the other hand, if $c_{h} \geq 0.20$ it is optimal to use the smallest value $q=250$.

Acknowledgments. The authors thank the associate editor and the referees for their careful reading of the manuscript and valuable suggestions, which have led to many improvements.

\section{References}

[1] Baron O (2010) Managing perishable inventories. Cochran JJ, ed. Wiley Encyclopedia of Operations Research and Management Science (John Wiley \& Sons, Hoboken, NJ).

[2] Baron O, Berman O, Perry D (2010) Continuous review inventory models for perishable items ordered in batches. Math. Meth. Oper. Res. 72:217-247.

[3] Berk E, Gürler U (2008) Analysis of the $(Q, r)$ inventory model for perishables with positive lead times and lost sales. Oper. Res. 56(5):1238-1246.

[4] Giri BC, Chaudhuri KS (1998) Deterministic models of perishable inventory with stock-dependent demand rate and nonlinear holding cost. Eur. J. Oper. Res 105:467-474.

[5] Graves S (1982) The application of queueing theory to continuous perishable inventory system. Management Sci. 28(4):400-406.

[6] Harris FW (1915) Operations and cost. Factory Management Series (Shaw, Chicago).

[7] Karaesmen I, Scheller-Wolf A, Deniz B (2010) Managing perishable and aging inventories: Review and future research directions. Kempf K, Keskinocak P, Uzsoy R, eds. Planning Production and Inventories in the Extended Enterprise, A State of the Art Handbook, Vol. 1 (Springer, New York), 393-436.

[8] Lian Z, Liu L (2001) Continuous review perishable inventory systems: Models and heuristics. IIE Trans. 33:809-822.

[9] Lian Z, Liu L, Neuts M (2005) A discrete-time model for common lifetime inventory systems. Math. Oper. Res. 30(3):718-732.

[10] Liu L, Lian Z (1999) $(s ; S)$ continuous review models for products with fixed lifetimes. Oper. Res. 47(1):150-158.

[11] Nahmias S (1982) Perishable inventory theory: A review. Oper. Res. 30(4):680-708.

[12] Nahmias S (2011) Perishable Inventory Systems (Springer, New York).

[13] Pal M (1989) The $(S-1, S)$ inventory model for deteriorating items with exponential lead time. Calcutta Statist. Assoc. Bull. 38:83-91.

[14] Perry D (1999) Analysis of a sampling control scheme for a perishable inventory system. Oper. Res. 47(6):966-973.

[15] Perry D, Stadje W, Zacks S (2005) Sporadic and continuous clearing policies for a production/inventory system under $M / G / \mathrm{demand}$. Math. Oper. Res. 30(2):354-368.

[16] Raafat F (1991) Survey of literature on continuously deteriorating inventory models. J. Oper. Res. Soc. 42:27-37.

[17] Rajan A, Steinberg R (1992) Dynamic pricing and ordering decisions by a monopolist. Management Sci. 38(2):240-262.

[18] Song Y, Lau HC (2004) A periodic-review inventory model with application to the continuous-review obsolescence problem. Eur. J. Oper. Res 159(1):110-120.

[19] Stadje W, Zacks S (2004) Upper first-exit times of compound Poisson processes revisited. Prob. Eng. Inf. Sci. 17:459-465.

[20] Weiss H (1980) Optimal ordering policies for continuous review perishable inventory models. Oper. Res. 28(2):365-374.

[21] Wilson RH (1934) A scientific routine for stock control. Harvard Bus. Rev. 13:116-128.

[22] Zacks S (2004) Generalized integrated telegrapher process and the distribution of related stopping times. J. Appl. Probab. 41:497-507.

[23] Zacks S (2005) Some recent results on the distributions of stopping times of compound Poisson processes with linear boundaries. J. Statist. Planning Inf. 130:95-109.

[24] Zipkin PH (2000) Foundations of Inventory Management (McGraw-Hill, New York). 